\title{
Prevalence of antifolate resistance mutations in Plasmodium falciparum isolates in Afghanistan
}

\author{
Ghulam R Awab ${ }^{1,4}$, Sasithon Pukrittayakamee ${ }^{2}$, Natsuda Jamornthanyawat ${ }^{3}$, Fazel Yamin ${ }^{4}$, Arjen M Dondorp ${ }^{1,5}$, \\ Nicholas PJ Day ${ }^{1,5}$, Nicholas J White ${ }^{1,5}$, Charles J Woodrow ${ }^{1,5}$ and Mallika Imwong ${ }^{3,6^{*}}$
}

\begin{abstract}
Background: Artesunate plus sulphadoxine-pyrimethamine (AS+SP) is now first-line treatment for Plasmodium falciparum infection in several south Asian countries, including Afghanistan. Molecular studies provide a sensitive means to investigate the current state of drug susceptibility to the SP component, and can also provide information on the likely efficacy of other potential forms of artemisinin-combination therapy.

Methods: During the years 2007 to 2010, 120 blood spots from patients with P. falciparum malaria were obtained in four provinces of Afghanistan. PCR-based methods were used to detect drug-resistance mutations in dhfr, dhps, pfcrt and pfmdr1, as well as to determine copy number of pfmdr1.

Results: The majority (95.5\%) of infections had a double mutation in the dhfr gene (C59R, S108N); no mutations at dhfr positions 16, 51 or 164 were seen. Most isolates were wild type across the dhps gene, but five isolates from the provinces of Kunar and Nangarhar in eastern Afghanistan had the triple mutation A437G / K540E / A581G; all five cases were successfully treated with three receiving AS+SP and two receiving dihydroartemisinin-piperaquine. All isolates showed the pfcrt SVNMT chloroquine resistance haplotype. Five of 79 isolates had the pfmdr1 N86Y mutation, while 52 had pfmdr1 Y184F; positions 1034, 1042 and 1246 were wild type in all isolates. The pfmdr 1 gene was not amplified in any sample.

Conclusions: This study indicates that shortly after the adoption of AS+SP as first-line treatment in Afghanistan, most parasites had a double mutation haplotype in dhfr, and a small number of isolates from eastern Afghanistan harboured a triple mutation haplotype in dhps. The impact of these mutations on the efficacy of AS+SP remains to be assessed in significant numbers of patients, but these results are clearly concerning since they suggest a higher degree of SP resistance than previously detected. Further focused molecular and clinical studies in this region are urgently required.
\end{abstract}

Keywords: Plasmodium falciparum, Malaria, Artemisinin combination therapy, Sulphadoxine-pyrimethamine, Dihydrofolate reductase, Dihydropteroate synthase

\section{Background}

Malaria remains a significant health concern in Afghanistan with ongoing transmission of both Plasmodium falciparum and Plasmodium vivax in many northern and eastern provinces [1-3]. Resistance of $P$. falciparum to chloroquine in Afghanistan was first apparent in the late 1980s [4], and by 2002 the clinical efficacy of chloroquine and amodiaquine

\footnotetext{
* Correspondence: noi@tropmedres.ac

${ }^{3}$ Department of Molecular Tropical Medicine and Genetics, Faculty of

Tropical Medicine, Mahidol University, Bangkok, Thailand

${ }^{6}$ Center for Emerging and Neglected Infectious Diseases, Mahidol University, Bangkok, Thailand

Full list of author information is available at the end of the article
}

in eastern Afghanistan was extremely low [5]; neither did the addition of artesunate to amodiaquine provide sufficient efficacy, being associated with an adequate clinical and parasitological response rate of only $72 \%$ [5]. During this period the efficacy of sulphadoxine-pyrimethamine (SP) was relatively preserved, with cure rates of $77-92 \%$ reported in eastern [5] and northern Afghanistan [6] as well as adjacent areas of Pakistan [7] (only the last study was able to correct for re-infection by PCR). Comparable success rates with SP were also noted in south-east Iran, although here there was also evidence of clinical resistance in a small number of parasites, associated with target mutations in

\section{Biomed Central}


dihydrofolate reductase (dhfr) and dihydropteroate synthase (dhps) [8]. The relative preservation of SP efficacy, along with increasing evidence for long-term benefits of artemisinin combination therapy (ACT), indicated that artesunate combined with sulphadoxine-pyrimethamine $(\mathrm{AS}+\mathrm{SP})$ would prove an effective regimen across the region. AS+SP became first-line treatment for P. falciparum infection in several south Asian countries, becoming first-line treatment in Afghanistan in 2004, followed by Iran (2006) and India and Pakistan (2007).

Molecular studies probably provide the earliest warning signs of resistance to the SP component of AS+SP $[9,10]$. For operational reasons, until recently there have been no molecular data on $P$. falciparum SP resistance markers in isolates obtained within the borders of Afghanistan. Indirect indicators of the likely situation in Afghanistan have been obtained through studies in areas of Pakistan with high influx rates of refugees from Afghanistan. Work in this context, undertaken in 20023 , indicated high efficacy for AS+SP with no evidence of worsening resistance in terms of molecular markers for SP [7].

Mutations in the $P$. falciparum chloroquine resistance transporter gene $(p f c r t)$ and multidrug resistance gene 1 (pfmdr1), as well as amplification of $p f m d r 1$, are associated with altered responses to a variety of antimalarials, including chloroquine as well as the ACT partner drugs amodiaquine, mefloquine and lumefantrine. This work provides an analysis of molecular markers of drug resistance to SP and other anti-malarials in isolates from several sites across Afghanistan.

\section{Methods}

Blood samples were obtained from patients presenting between July 2007 and February 2010 at malaria control centres in the provinces (towns) of Nangarhar (Jalalabad), Kunar (Asadabad), Faryab (Maimaneh) and Takhar (Taloqan). The patients were adults and children over six months old presenting with symptomatic, uncomplicated, microscopically confirmed mono-infection with asexual stages of $P$. falciparum. Blood samples were collected on filter paper (Whatman 3MM) at enrolment. Each filter paper was dried and individually stored in a plastic bag containing silica gel. All filter papers were subsequently transported to the Faculty of Tropical Medicine, Mahidol University, Bangkok, Thailand. Parasite DNA from dried blood spots was extracted via the QIAmp DNA Mini kit using the standard protocol and stored at $-20^{\circ} \mathrm{C}$ until use. PCRRFLP directed at specific mutations in dhfr, dhps, pfcrt, pfmdr1 as well as assessment of pfmdr1 copy number was undertaken using previously described techniques [11-14]; amplification was not always successful, reflecting differing sensitivities of each PCR.
In addition, samples with multiple mutations in dhps (according to RFLP) were sequenced with the primer corresponding to the published sequences R2 and R/ [11] to confirm multiple mutant genotypes. For the purposes of analysis, in five samples where mixed wild-type / mutant alleles were found at a single locus, the allele was classified as mutant.

The study was approved by the Ethics Committee of the Faculty of Tropical Medicine, Mahidol University, Thailand, the Oxford Tropical Research Ethics Committee, Oxford University, UK and the Institutional Review Board of the Afghan Public Health Institute, Ministry of Public Health, Afghanistan. The work described was undertaken within an overall framework of studies described at the clinicaltrials.gov website under NCT00682578.

\section{Results}

A total of 120 samples were collected, involving 83 patients in Nangarhar, 22 in Kunar, 11 in Takhar and four in Faryab provinces. Individual allele results are shown in Table 1 and Figure 1. More than 90\% of genotyped $P$. falciparum infections had a double mutation in the $d h f r$ gene (C59R, S108N). No mutations at $d h f r$ positions 16,51 or 164 were seen.

In the dhps gene, mutations were seen at A437 (7/57 isolates had $437 \mathrm{G}$ and one isolate from Faryab province had 437S), K540E (5/60) and A581G (8/60) (Figure 1A). The dhps mutations were clearly linked when examining haplotypes (Table 2), with K540E only seen when both

\begin{tabular}{|c|c|c|c|c|}
\hline Gene & Mutation examined & $\mathrm{N}$ & Mutated & (\%) \\
\hline \multirow[t]{5}{*}{$\overline{\mathrm{DHFR}}$} & A16V & 44 & 0 & (0) \\
\hline & N511 & 44 & 0 & (0) \\
\hline & C59R & 44 & 42 & $(95.5)$ \\
\hline & S108N & 44 & 44 & $(100)$ \\
\hline & I164L & 44 & 0 & (0) \\
\hline \multirow[t]{5}{*}{ DHPS } & $\mathrm{S} 436 \mathrm{~A}$ & 60 & 0 & (0) \\
\hline & $\mathrm{A} 437 \mathrm{G} / \mathrm{S}$ & 57 & $8^{*}$ & (14) \\
\hline & $\mathrm{K} 540 \mathrm{E}$ & 60 & 5 & $(8.3)$ \\
\hline & A581G & 60 & 8 & (13.3) \\
\hline & A613S & 60 & 0 & (0) \\
\hline \multirow[t]{6}{*}{ PFMDR1 } & N86Y & 79 & 5 & (6) \\
\hline & Y184F & 79 & 52 & $(65.8)$ \\
\hline & S1034C & 85 & 0 & (0) \\
\hline & N1042D & 80 & 0 & (0) \\
\hline & D1246Y & 43 & 0 & (0) \\
\hline & Copy number** & 62 & 0 & $(0)$ \\
\hline PFCRT & K76T & 49 & 49 & $(100)$ \\
\hline
\end{tabular}

*7 isolates had A437G and1 isolate had A437S; **Mutated = amplified. 


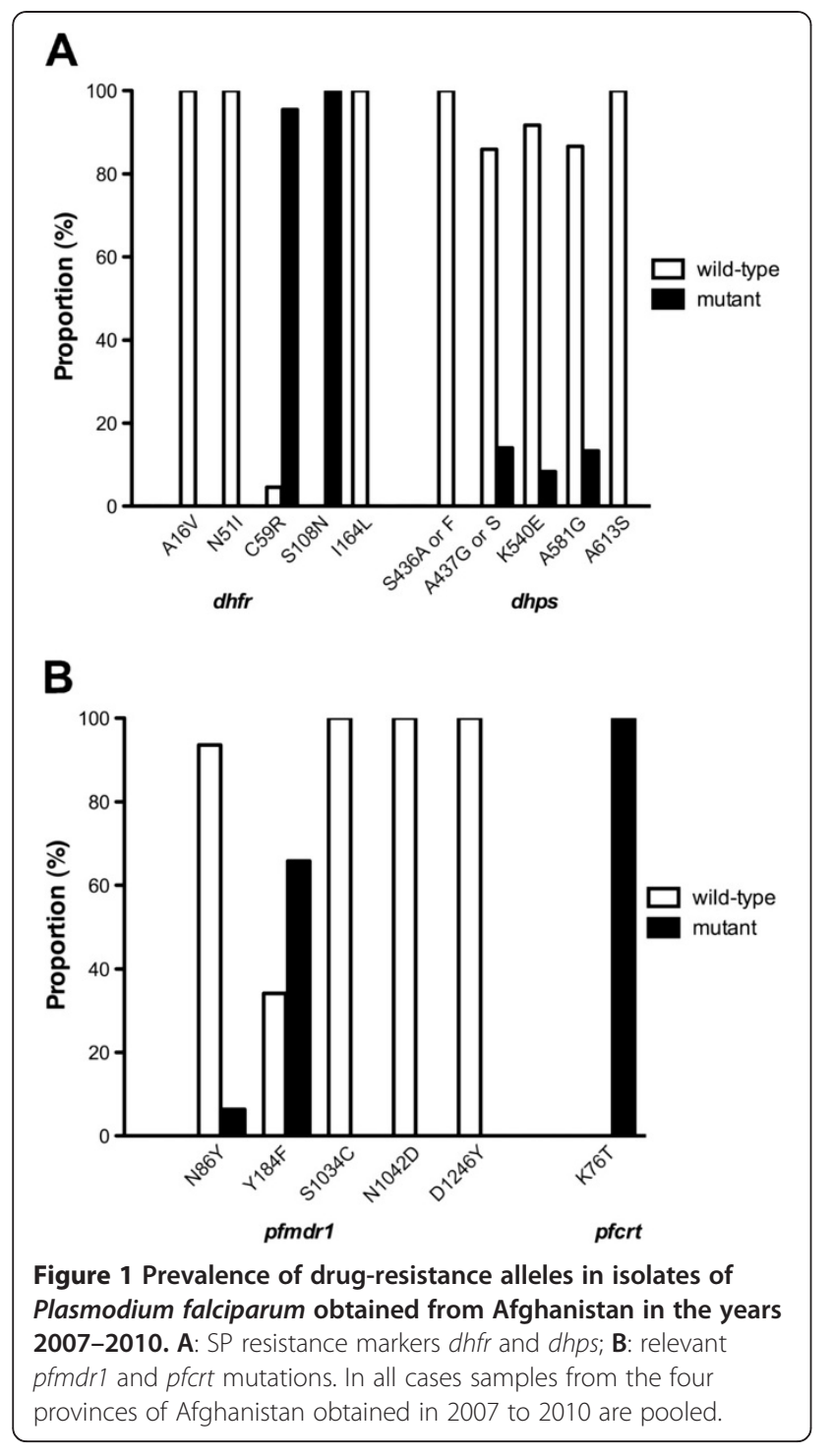

A437G and A581G were present, a similar pattern to that seen in sulphadoxine-resistant parasites from Southeast Asia $[15,16]$. Furthermore, all five isolates with the triple dhps mutant haplotype were obtained in eastern Afghanistan, being derived from Kunar (four isolates) and Nangarhar (one isolate). The patients harbouring these parasites were seen in late 2009 and treated with AS $+\mathrm{SP}$ (three cases) and dihydroartemisinin-piperaquine (two cases); all were followed to 42 days without experiencing clinical or parasitological recurrence.

All isolates showed the pfcrt K76T mutation with no mixed infections (Table 1, Figure 1B). Sequencing in a subset of 28 isolates confirmed the SVNMT pfcrt haplotype, consistent with previous studies of pfcrt from Afghanistan [17]. Five of 79 isolates had the pfmdr1 N86Y mutation, and 52/79 had pfmdr1 Y184F; positions 1034, 1042 and 1246 were wild type in all isolates. The pfmdr1 gene was not amplified in any sample.
Table $2 \mathrm{dhfr} / \mathrm{dhps}$ and pfmdr1 haplotypes

\begin{tabular}{|c|c|c|c|}
\hline \multirow{2}{*}{$\frac{\text { Genes }}{d h f r d h p s}$} & \multicolumn{2}{|c|}{ Haplotype } & \multirow{2}{*}{ Number of isolates } \\
\hline & ANCNI & ----- & \\
\hline & ANCNI & SAKAA & 1 \\
\hline & ANRNI & SAKAA & 21 \\
\hline & ANRNI & SGKAA & 1 \\
\hline & ANRNI & SSKGA & 1 \\
\hline & ANRN $\mid$ & SGEGA & 2 \\
\hline & ANRNI & $S-K A A$ & 2 \\
\hline & ANRN & $S-K \mathbf{G A}$ & 1 \\
\hline & ANRNI & ----- & 14 \\
\hline & ----- & SAKAA & 27 \\
\hline & ----- & SGKGA & 1 \\
\hline & ----- & SGEGA & 3 \\
\hline \multirow[t]{11}{*}{ pfmdr1 } & \multicolumn{2}{|c|}{ NYSND } & 16 \\
\hline & \multicolumn{2}{|c|}{ NYSN - } & 9 \\
\hline & \multicolumn{2}{|c|}{ NYS -- } & 2 \\
\hline & \multicolumn{2}{|c|}{ NFSND } & 23 \\
\hline & \multicolumn{2}{|c|}{ NFSN - } & 21 \\
\hline & \multicolumn{2}{|c|}{ NFS -- } & 3 \\
\hline & \multicolumn{2}{|c|}{ YFSND } & 2 \\
\hline & \multicolumn{2}{|c|}{ YFSN - } & 3 \\
\hline & \multicolumn{2}{|c|}{-- SND } & 2 \\
\hline & \multicolumn{2}{|c|}{$--S N-$} & 4 \\
\hline & \multicolumn{2}{|c|}{-----} & 5 \\
\hline
\end{tabular}

Haplotypes were determined by the allele at DHFR positions 16,51, 59, 108 and 164, DHPS positions 436, 437, 540, 581 and 613 and PFMDR1 positions 86 184, 1034, 1042 and 1246. Mutant alleles are indicated in bold.

\section{Discussion}

A regional overview of published $d h f r$ and dhps marker data from the last decade, including these new data from Afghanistan along with published data obtained in Iran, Pakistan and northern India (Table 3), reveals a broadly consistent picture (Figure 2). Two mutations in $d h f r$ (C59R and S108N) are at or close to fixation throughout the region, but few samples with triple mutations in $d h f r$ have been reported, and the critical $d h f$ I164L mutation has remained extremely rare. dhps mutations have generally been found only in a minority of samples, and generally as single mutations. However the A437G mutation has been widespread for a considerable time, with samples from southern [18] and north-western [19] Pakistan showing mutation frequencies of 25 to $60 \%$. Previous work in south-east Iran has additionally described relatively high numbers of isolates with S436A/F [8]. From the perspective of combined mutations in $d h f r$ and dhps, quintuple mutant haplotypes have remained very rare.

This study provides evidence for the presence of parasites with relatively higher levels of SP resistance in 
Table 3 Published molecular surveys of sulphadoxine markers in south-western Asia

\begin{tabular}{|c|c|c|c|c|}
\hline Country & State/province & Site on map & Reference & Year of sampling \\
\hline \multirow{4}{*}{ Afghanistan } & Nangarhar & 1 & \multirow{4}{*}{ This study } & \multirow{4}{*}{$2007-2010$} \\
\hline & Takhar & 2 & & \\
\hline & Faryab & 3 & & \\
\hline & Kunar & 4 & & \\
\hline \multirow{3}{*}{ Pakistan } & Khyber Pakhtunkhwa & 5 & [19] & 2007 \\
\hline & Khyber Pakhtunkhwa & 6 & {$[7]$} & $2000-2003$ \\
\hline & Baluchistan, Sindh & $7-9$ & [18] & 2005-2007 \\
\hline \multirow{7}{*}{ Iran } & \multirow{6}{*}{ Sistan and Baluchistan } & 10 & {$[20]$} & $2000-2002$ \\
\hline & & 11 & {$[20]$} & 2004 \\
\hline & & 12 & [21] & 2003-2004 \\
\hline & & 13 & {$[22]$} & $2005-2008$ \\
\hline & & 14 & [23] & 2008-2010 \\
\hline & & 15 & {$[8]$} & 2003-2005 \\
\hline & Sistan and Baluchistan, Hormuzgan, Kerman & 16 & [24] & $2000-2001$ \\
\hline \multirow{2}{*}{ India } & Rajasthan & 17 & [25] & Not stated \\
\hline & Delhi & 18 & [26] & 1995-2001 \\
\hline
\end{tabular}

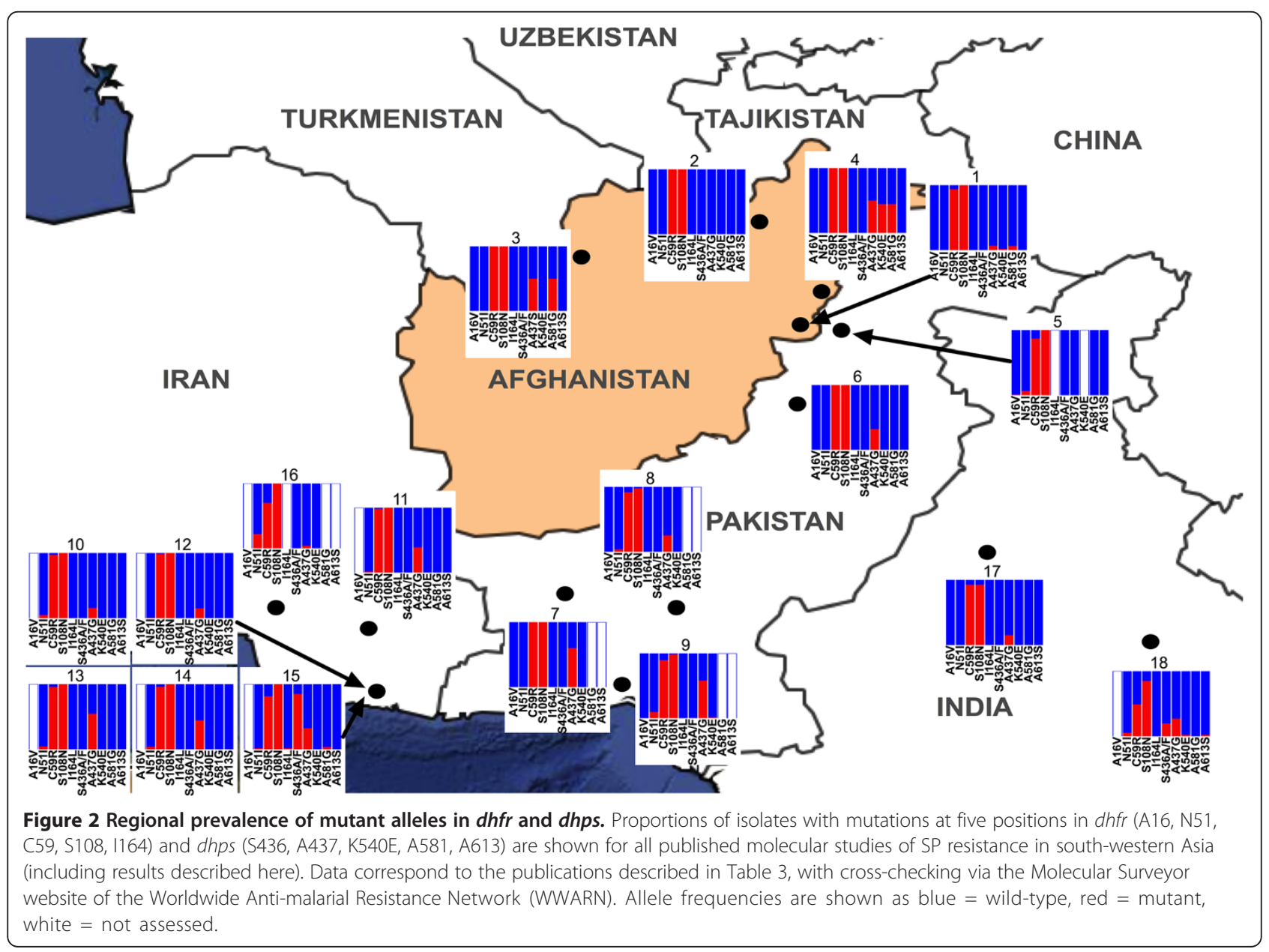


eastern Afghanistan, and suggests that molecularly determined SP resistance may be close to compromising the efficacy of first-line treatment for falciparum malaria in Afghanistan. Development of this level of resistance was presumably brought about by the long-term use of $\mathrm{SP}$ in the region, since at the time of collection, artemisinin combination therapy $(\mathrm{AS}+\mathrm{SP})$ had only just become first-line treatment for falciparum malaria.

There are few data on the clinical response to AS+SP in the setting of double $d h f r$ and triple dhps mutations, and relatively few patients documented as having this haplotype have entered prospective clinical trials. It is therefore difficult to predict the likely evolution of drug resistance alleles in this area, and much may depend on the field implementation of AS+SP. Unfortunately, there is evidence that inappropriate anti-malarial regimens continue to be prescribed in Afghanistan, including SP alone [27]. This is likely to lead to a further worsening of SP resistance, for example via mutations in dhfr (particularly I164L), and an AS+SP combination that increasingly resembles artesunate monotherapy (with the additional risk of emerging artemisinin resistance). In an alternative scenario, consistent use of AS+SP holds the potential to prevent an increase in (or even reduce) the prevalence of SP-resistant parasites, in an analogous manner to that seen for mefloquine [28] and generally predicted for artemisinin combination partners [29]. This type of development was documented in south-east Iran, where the introduction of $\mathrm{AS}+\mathrm{SP}$ appears to have prevented SP resistance from worsening, and led to a reduction in the proportion of parasites with the $437 \mathrm{G}$ dhps mutation [23]. Whatever the relative probabilities of these two outcomes, ongoing studies examining molecular markers as well as clinical efficacy, both in Afghanistan and more widely across the region, are urgently needed.

\section{Conclusions}

Molecular data reveal the presence in eastern Afghanistan of $P$. falciparum parasites with higher levels of SP resistance than previously documented in southern Asia. There is no evidence yet that this is compromising the efficacy of first-line treatment for falciparum malaria in Afghanistan (AS+SP), but ongoing studies examining molecular markers and clinical trials will continue to be important tools in monitoring efficacy, both in Afghanistan and more widely across the region.

\section{Competing interests}

The authors declare that they have no competing interests.

\section{Authors' contributions}

GRA, SP, AD, ND, NW, CW and MI participated in the conception and design of the study. GRA and FY were responsible for obtaining samples and supervision of study sites. NJ and MI carried out the molecular biology. GRA, $\mathrm{CW}$ and $\mathrm{MI}$ were responsible for data analysis, data management and drafting of the manuscript. All authors read and approved the final manuscript.

\section{Acknowledgements}

GRA was supported by the Mahidol-Oxford Research Unit as a continuation of an MSc scholarship granted by the Thailand International Development and Cooperation Agency. Ml is supported by Mahidol University and The Dean's Research Fund, Faculty of Tropical Medicine. The study was supported by the Li Ka Shing Foundation and was part of the Wellcome Trust Mahidol University-Oxford Tropical Medicine Research Programme supported by the Wellcome Trust of Great Britain.

The investigators are grateful to the doctors, laboratory technicians and malaria workers in all four sites in Afghanistan, the Afghanistan National Malaria and Leishmania Control Programme (NMLCP), the four provincial health directorates and the senior management of the Ministry of Public Health and WHO-Afghanistan.

\section{Author details}

${ }^{1}$ Mahidol-Oxford Tropical Medicine Research Unit (MORU), Faculty of Tropical Medicine, Bangkok, Thailand. 'Department of Clinical Tropical Medicine, Faculty of Tropical Medicine, Mahidol University, Bangkok, Thailand. ${ }^{3}$ Department of Molecular Tropical Medicine and Genetics, Faculty of Tropical Medicine, Mahidol University, Bangkok, Thailand. ${ }^{4}$ Ministry of Public Health, Islamic Republic of Afghanistan, Kabul, Afghanistan. ${ }^{5}$ Centre for Clinical Vaccinology and Tropical Medicine, Churchill Hospital, University of Oxford, Oxford, UK. ${ }^{6}$ Center for Emerging and Neglected Infectious Diseases, Mahidol University, Bangkok, Thailand.

Received: 24 October 2012 Accepted: 11 March 2013

Published: 15 March 2013

\section{References}

1. Leslie T, Mohammed N, Omar H, Rasheed HU, Vorst F, Sediqi AM: Malaria sentinel surveillance in Afghanistan. Afghanistan Annual Malaria Journal 2008:114-128.

2. Gething PW, Patil AP, Smith DL, Guerra CA, Elyazar IR, Johnston GL, Tatem AJ, Hay SI: A new world malaria map: Plasmodium falciparum endemicity in 2010. Malar J 2011, 10:378.

3. WHO: Afghanistan country profile. 2011. http://www.who.int/malaria/ publications/country-profiles/profile_afg_en.pdf.

4. Delfini LF: The first case of Plasmodium falciparum resistant to chloroquine treatment discovered in the Republic of Afghanistan. Trans R Soc Trop Med Hyg 1989, 83:316.

5. Durrani N, Leslie T, Rahim S, Graham K, Ahmad F, Rowland M: Efficacy of combination therapy with artesunate plus amodiaquine compared to monotherapy with chloroquine, amodiaquine or sulfadoxinepyrimethamine for treatment of uncomplicated Plasmodium falciparum in Afghanistan. Trop Med Int Health 2005, 10:521-529.

6. Ezard N, Nellepalli P, Asha AW: Sulfadoxine-pyrimethamine remains efficacious against uncomplicated, Plasmodium falciparum malaria in north-eastern Afghanistan. Ann Trop Med Parasitol 2004, 98:85-88.

7. Kolaczinski K, Leslie T, Ali I, Durrani N, Lee S, Barends M, Beshir K, Ord R, Hallett R, Rowland M: Defining Plasmodium falciparum treatment in South West Asia: a randomized trial comparing artesunate or primaquine combined with chloroquine or SP. PLoS One 2012, 7:e28957.

8. Heidari A, Dittrich S, Jelinek T, Kheirandish A, Banihashemi K, Keshavarz H: Genotypes and in vivo resistance of Plasmodium falciparum isolates in an endemic region of Iran. Parasitol Res 2007, 100:589-592.

9. Gregson A, Plowe CV: Mechanisms of resistance of malaria parasites to antifolates. Pharmacol Rev 2005, 57:117-145.

10. Laufer MK, Djimde AA, Plowe CV: Monitoring and deterring drug-resistant malaria in the era of combination therapy. Am J Trop Med Hyg 2007, 77:160-169.

11. Duraisingh MT, Curtis J, Warhurst DC: Plasmodium falciparum: detection of polymorphisms in the dihydrofolate reductase and dihydropteroate synthetase genes by PCR and restriction digestion. Exp Parasitol 1998, 89:1-8.

12. Duraisingh MT, Jones $P$, Sambou I, von Seidlein L, Pinder M, Warhurst DC: The tyrosine-86 allele of the pfmdr 1 gene of Plasmodium falciparum is associated with increased sensitivity to the anti-malarials mefloquine and artemisinin. Mol Biochem Parasitol 2000, 108:13-23. 
13. Price R, Robinson G, Brockman A, Cowman A, Krishna S: Assessment of pfmdr 1 gene copy number by tandem competitive polymerase chain reaction. Mol Biochem Parasitol 1997, 85:161-169.

14. Carrara VI, Zwang J, Ashley EA, Price RN, Stepniewska K, Barends M, Brockman A, Anderson T, McGready R, Phaiphun L, Proux S, van Vugt M, Hutagalung R, Lwin KM, Phyo AP, Preechapornkul P, Imwong M, Pukrittayakamee S, Singhasivanon P, White NJ, Nosten F: Changes in the treatment responses to artesunate-mefloquine on the northwestern border of Thailand during 13 years of continuous deployment. PLoS One 2009, 4:e4551.

15. Vinayak S, Alam MT, Mixson-Hayden T, McCollum AM, Sem R, Shah NK, Lim P, Muth S, Rogers WO, Fandeur T, Barnwell JW, Escalante AA, Wongsrichanalai C, Ariey F, Meshnick SR, Udhayakumar V: Origin and evolution of sulfadoxine resistant Plasmodium falciparum. PLoS Pathog 2010, 6:e1000830.

16. Lumb V, Das MK, Singh N, Dev V, Khan W, Sharma YD: Multiple origins of Plasmodium falciparum dihydropteroate synthetase mutant alleles associated with sulfadoxine resistance in India. Antimicrob Agents Chemother 2011, 55:2813-2817.

17. Beshir K, Sutherland CJ, Merinopoulos I, Durrani N, Leslie T, Rowland M, Hallett RL: Amodiaquine resistance in Plasmodium falciparum malaria in Afghanistan is associated with the pfcrt SVMNT allele at codons 72 to 76. Antimicrob Agents Chemother 2010, 54:3714-3716.

18. Ghanchi NK, Ursing J, Beg MA, Veiga MI, Jafri S, Martensson A: Prevalence of resistance associated polymorphisms in Plasmodium falciparum field isolates from southern Pakistan. Malar J 2011, 10:18

19. Khatoon L, Baliraine FN, Bonizzoni M, Malik SA, Yan G: Prevalence of antimalarial drug resistance mutations in Plasmodium vivax and P. falciparum from a malaria-endemic area of Pakistan. Am J Trop Med Hyg 2009, 81:525-528.

20. Zakeri S, Afsharpad M, Raeisi A, Djadid ND: Prevalence of mutations associated with antimalarial drugs in Plasmodium falciparum isolates prior to the introduction of sulphadoxine-pyrimethamine as first-line treatment in Iran. Malar J 2007, 6:148.

21. Zakeri S, Gil JP, Bereckzy S, Djadid ND, Bjorkman A: High prevalence of double Plasmodium falciparum dhfr mutations at codons 108 and 59 in the Sistan-Baluchistan province, Iran. J Infect Dis 2003, 187:1828-1829.

22. Zakeri S, Farahani MS, Afsharpad M, Salehi M, Raeisi A, Djadid ND: High prevalence of the $437 \mathrm{G}$ mutation associated with sulfadoxine resistance among Plasmodium falciparum clinical isolates from Iran, three years after the introduction of sulfadoxine-pyrimethamine. Int I Infect Dis 2010, 14(Suppl 3):e123-e128.

23. Afsharpad M, Zakeri S, Pirahmadi S, Djadid ND: Molecular monitoring of Plasmodium falciparum resistance to antimalarial drugs after adoption of sulfadoxine-pyrimethamine plus artesunate as the first line treatment in Iran. Acta Trop 2012, 121:13-18.

24. Jafari S, Le Bras J, Asmar M, Durand R: Molecular survey of Plasmodium falciparum resistance in south-eastern Iran. Ann Trop Med Parasitol 2003, 97:119-124.

25. Garg S, Saxena V, Kanchan S, Sharma P, Mahajan S, Kochar D, Das A: Novel point mutations in sulfadoxine resistance genes of Plasmodium falciparum from India. Acta Trop 2009, 110:75-79.

26. Ahmed A, Bararia D, Vinayak S, Yameen M, Biswas S, Dev V, Kumar A, Ansari MA, Sharma YD: Plasmodium falciparum isolates in India exhibit a progressive increase in mutations associated with sulfadoxinepyrimethamine resistance. Antimicrob Agents Chemother 2004, 48:879-889.

27. Leslie T, Mikhail A, Mayan I, Anwar M, Bakhtash S, Nader M, Chandler C, Whitty $\mathrm{CJ}$, Rowland M: Overdiagnosis and mistreatment of malaria among febrile patients at primary healthcare level in Afghanistan: observational study. BMJ 2012, 345:e4389.

28. Nosten F, van Vugt M, Price R, Luxemburger C, Thway KL, Brockman A, McGready R, ter Kuile F, Looareesuwan S, White NJ: Effects of artesunatemefloquine combination on incidence of Plasmodium falciparum malaria and mefloquine resistance in western Thailand: a prospective study. Lancet 2000, 356:297-302.

29. White NJ: Antimalarial drug resistance. J Clin Invest 2004, 113:1084-1092.

doi:10.1186/1475-2875-12-96

Cite this article as: Awab et al:: Prevalence of antifolate resistance mutations in Plasmodium falciparum isolates in Afghanistan. Malaria Journal 2013 12:96.

\section{Submit your next manuscript to BioMed Central and take full advantage of:}

- Convenient online submission

- Thorough peer review

- No space constraints or color figure charges

- Immediate publication on acceptance

- Inclusion in PubMed, CAS, Scopus and Google Scholar

- Research which is freely available for redistribution 